\title{
Vigilância nas nuvens: relato de experiência de acadêmicos do PET- Saúde/Interprofissionalidade
}

\author{
Surveillance in the clouds: experience report of PET students- Health/Interprofessionality \\ Vigilancia en las nubes: informe de experiencia de estudiantesde PET- Salud/ \\ Interprofesionalidad
}

Larissa Tolentino Lôpo ${ }^{1 *}$, Adriana Barbosa Rodrigues ${ }^{1}$, Ana Rúbia Rockenbach ${ }^{1}$, Fernanda Santos Noronha ${ }^{1}$, Lucas de Andrade Huber ${ }^{1}$, Luis Eugênio Gomes Freitas ${ }^{1}$, Vítor Fonseca Bastos $^{1}$, Ana Paula Figueiredo Guimarães de Almeida ${ }^{1}$, Claudia Danyella Alves Leão Ribeiro ${ }^{1}$, Aline Soares Figueiredo Santos ${ }^{1}$.

\section{RESUMO}

Objetivo: Relatar a experiência dos acadêmicos do PET-Saúde/Interprofissionalidade na implantação da vigilância nas nuvens, com dados de pacientes portadores de hipertensão arterial e diabetes mellitus, cadastrados em equipes da Estratégia de Saúde da Família. Relato de experiência: Trata-se de um estudo descritivo, na modalidade relato de experiência, de acadêmicos de medicina, enfermagem e odontologia. Foi elaborada uma planilha no programa Excel e preenchida com os dados referentes às condições de saúde de usuários acompanhados. As informações foram coletadas em prontuários e exames laboratoriais. Os usuários foram estratificados com risco cardiovascular baixo, médio, alto e muito alto. A planilha foi salva no formato on-line, de maneira que todos os profissionais tenham acesso fácil e simultâneo. Considerações finais: A vigilância nas nuvens permite a identificação imediata de usuários em risco, propiciando ações direcionadas para um melhor cuidado. A experiência apontou a necessidade do registro adequado de informações sobre os pacientes, além de sua atualização periódica, a fim de ser um instrumento de fácil e confiável acesso para acompanhamento dos usuários e, portanto, da prática da vigilância em saúde, permitindo um monitoramento mais efetivo, diminuição do risco de complicações e melhoria da qualidade de vida.

Palavras-chave: Vigilância de doença crônica, Hipertensão, Diabetes, Atenção primária à saúde.

\begin{abstract}
Objective: To report the experience of PET-Saúde/Interprofessional students in the implementation of surveillance in the clouds, with data from patients with arterial hypertension and diabetes mellitus registered in the Family Health Strategy. Experience report: This is a descriptive study in the experience report mode of medical, nursing and dentistry students. A spreadsheet was prepared in the Excel program, and filled with data from patients with the aforementioned diseases. Information was collected from medical records and laboratory tests. Patients were stratified with low, medium, high and very high cardiovascular risk. The spreadsheet was saved in the online format, so that all professionals have simultaneous access. Final considerations: Cloud surveillance allows immediate identification of patients at risk, providing actions aimed at better care. The experience pointed out the need for adequate registration of information about patients, in addition to their periodic updating, in order to be an easy and reliable instrument for monitoring users and, therefore, the practice of health surveillance, allowing for more effective monitoring, decreased risk of complications and improved quality of life.
\end{abstract}

Keywords: Chronic disease indicators, Hypertension, Diabetes, Primary health care.

1 Universidade Estadual de Montes Claros (UNIMONTES), Montes Claros - MG.

*E-mail: larissatolentinolopo@gmail.com

SUBMETIDO EM: 1/2021

ACEITO EM: 2/2021

PUBLICADO EM: 4/2021 


\section{RESUMEN}

Objetivo: Informar la experiencia de los estudiantes de PET-Saúde / Interprofesional en la implementación de la vigilancia en las nubes, con datos de pacientes con hipertensión arterial y diabetes mellitus registrados en la Estrategia de Salud Familiar. Informe de experiencia: este es un estudio descriptivo sobre el informe de experiencia de estudiantes de medicina, enfermería y odontología. Se preparó una hoja de cálculo en el programa Excel y se completó con datos de pacientes con las enfermedades mencionadas. Se recopiló información de registros médicos y pruebas de laboratorio. Los pacientes fueron estratificados con riesgo cardiovascular bajo, medio, alto y muy alto. La hoja de cálculo se guardó en un formato en línea, para que todos los profesionales tengan acceso simultáneo. Consideraciones finales: la vigilancia en la nube permite la identificación inmediata de pacientes en riesgo, proporcionando acciones dirigidas a una mejor atención. La experiencia señaló la necesidad de un registro adecuado de la información sobre los pacientes, además de actualizaciones periódicas, para ser un instrumento fácil y confiable para monitorear a los usuarios y, por lo tanto, la práctica de la vigilancia de la salud, lo que permite un monitoreo más efectivo, disminución del riesgo de complicaciones y mejora de la calidad de vida.

Palabras clave: Indicadores de enfermedades crónicas, Hipertensión, Diabetes, Atención primaria de salud.

\section{INTRODUÇÃO}

O Programa de Educação pelo Trabalho para a Saúde (PET-Saúde) foi instituído pelo Ministério da Saúde em 2008, com intuito de proporcionar aquisição de habilidades e qualificação dos processos de integração ensino-serviço-comunidade, de forma articulada entre o Sistema Único de Saúde (SUS) e as instituições de ensino superior (SILVA RP, et al., 2015; BRASIL, 2019; GARCIA SO, et al., 2019; ALMEIDA RGS, et al., 2019).

Em 2018 foi lançado o PET-Saúde/Interprofissionalidade, que envolve atores do SUS: profissionais de saúde, gestores e usuários; bem como da comunidade acadêmica, como professores e estudantes. Considera ações a serem desenvolvidas na Atenção Primária à Saúde (APS) para a qualificação dos futuros profissionais que, numa prática colaborativa, poderão obter respostas mais efetivas na melhoria da atenção à saúde (STARFIELD B, 2002; REEVES S, et al., 2016; BATISTA NA, et al., 2018; BRASIL, 2018; PEREIRA MF, 2018; BRASIL, 2019).

Dentre as áreas estratégicas para o cuidado na APS estão ações que priorizam o cuidado de usuários com Doenças Crônicas Não Transmissíveis (DCNT), que são responsáveis por cerca de $70 \%$ de todas as mortes no mundo (SILOCCHI C e JUNGUES JR, 2017). Estudos indicam que o aumento das DCNT se dá em virtude de fatores de risco como tabagismo, inatividade física, alcoolismo e alimentação não saudável. Tais doenças têm se tornado prioridade para o sistema de saúde no Brasil, na medida em que representam causas importantes de morbimortalidade (MALTA DC, et al., 2017; TANAKA OY, et al., 2019; SANTOS MPR dos, et al., 2020).

Dessa forma, faz parte do processo de trabalho dos profissionais de saúde, das equipes da Estratégia Saúde da Família (ESF), acompanhar na APS condições crônicas como Hipertensão Arterial Sistêmica (HAS) e Diabetes Mellitus (DM), estimulando mudança de comportamento e estilo de vida saudável dos usuários, apoiando o autocuidado, além de tratar e rastrear as complicações (MENDES EV, 2012; MINAS GERAIS, 2013; BRASIL, 2017). Para isso, é necessário conhecer, estratificar o risco e realizar vigilância em saúde, como estratégias capazes de direcionar ações que contribuirão efetivamente nesse cuidado (MINAS GERAIS, 2013; MALTA DC, et al., 2015).

Nesse sentido, a estratificação do risco cardiovascular (RCV) tem como vantagens mensurar o RCV global e nortear o acompanhamento desses fatores de risco pelos profissionais de saúde (MINAS GERAIS, 2013). A doença cardiovascular (DCV) é um problema de saúde pública nas sociedades modernas, sendo a principal causa de morbimortalidade no mundo e no Brasil (SOCERJ, 2017). Dados de 2013 registram 339 672 óbitos decorrentes de DCV, sendo responsável por duas vezes mais mortes que todos os tipos de câncer juntos, duas vezes e meia mais que todos os acidentes e mortes violentas e ainda seis vezes mais que infecções (SOCERJ, 2017). 
Nesse contexto, a estratificação do RCV permite que as equipes da APS possam acompanhar cada paciente e garantir um acompanhamento e cuidado que realmente abranjam suas necessidades. Possibilita ainda, prestar uma assistência de excelência, a fim de melhorar os indicadores de saúde da população adscrita, com consequente melhoria da qualidade de vida das pessoas (MINAS GERAIS, 2013).

Sendo assim, a disponibilização dos dados de estratificação de hipertensos e diabéticos, por meio da vigilância nas nuvens é uma técnica inovadora que utiliza dados on-line para permitir o fácil acesso à informação, de forma dinâmica e compartilhada. O sistema baseia-se na utilização de planilha virtual de acompanhamento dos usuários que precisam de monitoramento (UNAS-SUS, 2017). Tal ferramenta possibilita que a informação seja armazenada de forma confiável, garantindo ainda o registro da classificação de risco e a comunicação instantânea entre os profissionais de saúde (ROJAS A, et al., 2016).

Por ser uma prática recente, muitas equipes da APS ainda não têm em seu processo de trabalho a utilização dessa rotina. Dessa forma, esse trabalho tem como objetivo relatar a experiência vivenciada por acadêmicos do PET-Saúde/Interprofissionalidade na implantação da vigilância nas nuvens, de pacientes portadores de HAS e DM.

\section{RELATO DE EXPERIÊNCIA}

Trata-se de um estudo descritivo, na modalidade de relato de experiência, realizado pelos acadêmicos de enfermagem, medicina e odontologia, participantes do PET-Saúde/Interprofissionalidade. A população alvo da estratificação de risco cardiovascular foram todos os portadores de HAS e DM, cadastrados em três equipes da ESF.

Inicialmente foi realizado um levantamento nas equipes de saúde com intuito de identificar as principais dificuldades relacionadas ao rastreamento e acompanhamento de pacientes com risco cardiovascular. A partir disso, foram pensadas estratégias e ferramentas que pudessem facilitar 0 rastreamento, acompanhamento, consulta e atualização de informações sobre os pacientes hipertensos e diabéticos e que fossem de acesso simultâneo.

Para tanto foi construída uma planilha utilizando o programa Microsoft Excel®, que disponibiliza campos para preenchimento de informações relacionadas à estratificação e acompanhamento dos pacientes hipertensos e diabéticos, por todos os profissionais, a partir de diversos equipamentos (computador, celular, tablet, notebook), conectados à internet.

Tal planilha foi composta pelos seguintes dados: data da estratificação de risco cardiovascular, dados de identificação (nome, gênero, data de nascimento, idade, endereço, número da família, número do prontuário, número da microárea), dados antropométricos (altura, peso, Índice de Massa Corporal (IMC) e circunferência abdominal), situação de tabagismo, condições para manter o autocuidado, identificação de HAS tratada ou não, DM, doença renal crônica, colesterol total, lipoproteína de alta densidade (HDL), campo de observações, data da última consulta, classificação do RCV (baixo, médio, alto ou muito alto) e campo para programação de exames (Quadro 1).

Os dados que compõem a planilha estão de acordo com a Linha Guia, como sendo necessárias para identificação do RCV dos pacientes (MINAS GERAIS, 2013). As pontuações que mostravam o grau de RCV apresentado por cada paciente foram obtidas por meio do Escore de Framingham (EF) e, em cada prontuário, foi anexado um instrumento para preenchimento do conjunto de dados obtidos.

As planilhas foram individualizadas para cada equipe de saúde, para fins organizacionais, sendo inseridas abas no Microsoft Exce/ $\Theta$, identificando cada equipe. As microáreas foram informadas em uma coluna específica contida na planilha, considerando a possibilidade de busca e/ou consulta por meio da ferramenta de filtro.

A etapa seguinte constituiu-se em coletar os dados diretamente nos prontuários físicos de cada paciente. Para tanto, foram identificados os pacientes hipertensos e diabéticos da área de abrangência diretamente com as equipes de saúde, que também disponibilizaram suas listas com o nome dos pacientes cadastrados no Programa Nacional de Hipertensão e Diabetes Mellitus (HIPERDIA) do SUS, facilitando a localização dos prontuários. 
As informações coletadas foram inseridas nessa planilha, sendo salvas nas nuvens, por meio de Planilhas Google. Dessa forma, foi permitido acesso on-line e simultâneo, tanto pelos acadêmicos, quanto pelos membros da equipe de saúde, por meio de senhas, de modo que as informações pudessem ser atualizadas instantaneamente, durante $o$ atendimento ao paciente.

Posteriormente, propôs-se a realização de Educação Permanente em Saúde (EPS) dos profissionais das equipes da ESF, de modo a sensibilizá-los e orientá-los, quanto ao uso da planilha nas nuvens como ferramenta facilitadora no acompanhamento e rastreamento dos pacientes hipertensos e diabéticos, para avaliação do RCV. A capacitação presencial perpassou pela apresentação das formas de acesso à planilha on-line, a interface nas nuvens, informações presentes na ferramenta, bem como sistemas de filtro de dados e outros aspectos relacionados ao acompanhamento de saúde.

O próximo passo planejado para o processo de implantação será o acompanhamento e avaliação da equipe de saúde em relação ao uso da vigilância nas nuvens, enquanto ferramenta de monitoramento dos pacientes de acordo com a estratificação de risco.

Quadro 1 - Dados dos pacientes a serem coletados na planilha de vigilância nas nuvens em Excel, 2021.

\begin{tabular}{|c|c|c|c|c|c|c|c|c|}
\hline \multicolumn{9}{|c|}{ Identificação } \\
\hline Data & Nome & Gênero & Endereço & $\mathrm{DN}^{*}$ & Idade & $\begin{array}{l}N^{\circ} \text { da } \\
\text { família }\end{array}$ & $\begin{array}{c}\mathrm{N}^{\circ} \text { do } \\
\text { prontuário }\end{array}$ & Microárea \\
\hline \multicolumn{9}{|c|}{ Dados Antropométricos/ Hábitos/ Condições sistêmicas } \\
\hline $\begin{array}{l}\text { Altura } \\
(\mathrm{m})\end{array}$ & $\begin{array}{l}\text { Peso } \\
(\mathrm{Kg})\end{array}$ & $\mathrm{IMC}^{* *}$ & $\mathrm{CA}^{* * *}$ & $\begin{array}{c}\text { Tabagista } \\
\text { (sim ou } \\
\text { não) }\end{array}$ & Autocuidado & $\begin{array}{l}\text { HAS } \\
\text { tratada }\end{array}$ & $\begin{array}{l}\text { HAS não } \\
\text { tratada }\end{array}$ & $\begin{array}{l}\text { Lesão de } \\
\text { órgão alvo }\end{array}$ \\
\hline \multicolumn{9}{|c|}{ Condições sistêmicas/ Exames laboratoriais } \\
\hline \multicolumn{3}{|c|}{$\begin{array}{c}\text { Diabetes mellitus } \\
\text { (sim ou não) }\end{array}$} & \multicolumn{2}{|c|}{$\begin{array}{l}\text { Lesão de órgão alvo } \\
\text { (sim ou não) }\end{array}$} & Colesterol total & \multicolumn{3}{|c|}{$\mathrm{HDL}^{* * * * *}$} \\
\hline \multicolumn{9}{|c|}{ Risco Cardiovascular } \\
\hline & Baixo & & Moderado & & Alto & & Muito Altc & \\
\hline
\end{tabular}

Legenda: ${ }^{\star} \mathrm{DN}$ : Data de Nascimento; ${ }^{* *} \mathrm{IMC}$ : Índice de Massa Corporal; ${ }^{* * *} \mathrm{CA}$ : Circunferência Abdominal;

${ }^{* \star \star *}$ HAS: Hipertensão Arterial Sistêmica; ${ }^{* * * * H D L: ~ H i g h ~ D e n s i t y ~ L i p o p r o t e i n s ~ o u ~ L i p o p r o t e i ́ n a s ~ d e ~ a l t a ~}$ densidade. Fonte: Lôpo LT, et al., 2021.

\section{DISCUSSÃO}

O grupo de acadêmicos PET-Saúde/Interprofissionalidade contribuiu com a estratificação de risco cardiovascular dos usuários com as principais situações crônicas causadoras de morbimortalidade na população da área de responsabilidade. Tal ação vem ao encontro da orientação que os participantes do PET devem problematizar as diversas demandas da população adscrita, levando em consideração seu contexto social e as necessidades de saúde mais frequentes (ALMEIDA RGS, et al., 2019).

A estratificação do RCV é de suma importância, uma vez que avalia o paciente com DCNT, auxiliando na redução de agravos e mortalidade (MENDEZ RDR, et al., 2018). Tal estratificação é uma forma de vigilância em saúde, pois visa o acompanhamento integral, buscando melhorar a qualidade de vida dos pacientes (DANTAS RCO e RONCALLI AG, 2019). Essa ação vai ao encontro do Plano de Ações Estratégicas para o Enfrentamento das DCNT e tem incentivado que os serviços públicos de saúde implementem ações de promoção, prevenção e controle, com um cuidado privilegiado de usuários com tais doenças na rede de atenção à saúde (BRASIL, 2013).

A estratificação e a vigilância em saúde nas nuvens, aqui relatadas, têm como vantagens a mensuração do RCV em tempo real, o rastreio das complicações, análise do prognóstico, bem como a capacidade de nortear o acompanhamento dos fatores de risco (MINAS GERAIS, 2013). É uma técnica inovadora que utiliza o acesso on-line aos dados, contribuindo para a melhoria dos serviços de saúde, e aperfeiçoando o acompanhamento da população adscrita, uma vez que a vigilância em saúde é o conjunto de atividades que ordena e articula elementos e informações sobre a situação de saúde, para enfrentar os problemas encontrados no território (SOARES CHA e LINHARES MSC, 2008; MINAS GERAIS, 2013). 
A metodologia de vigilância nas nuvens permitirá que todos os profissionais de saúde acessem as informações e avaliem as pessoas hipertensas e diabéticas da ESF, possibilitando o acompanhamento adequado dos usuários de acordo com seu grau de RCV - baixo, médio, alto e muito alto. A estratificação de risco considerou as recomendações da linha-guia de HAS, DM e doença renal crônica da Secretaria de Estado de Saúde de Minas Gerais (MINAS GERAIS, 2013). O Escore de Risco de Framingham é recomendado pelo Ministério da Saúde para detecção e estimativa de RCV total, sendo de grande relevância na prevenção de tais condições (OLIVEIRA SX, et al., 2018).

A elaboração da planilha otimizará o trabalho da equipe já que, por meio dela, as principais falhas no registro dos atendimentos poderão ser minimizadas, pois a vigilância nas nuvens permitirá à equipe criar novas rotinas e padronizar informações necessárias para o adequado monitoramento, estratificação do risco e acompanhamento dos diabéticos e hipertensos, objetivando alcançar os melhores resultados.

Resultados de estudo trazem que a planilha de vigilância nas nuvens é uma ferramenta que possibilita priorizar a integração do cuidado, enxergar o sujeito em sua totalidade e não somente a sua patologia; contrapondo-se às antigas prioridades do sistema de saúde que se direcionavam para as condições agudas (DA SILVA LG, et al., 2018). Dessa forma, é possível ter mais informações sobre a evolução e história do paciente para, a partir delas, tomar atitudes visando reduzir as consequências da evolução insidiosa das doenças crônicas, como é o caso da HA e DIA.

Após a implementação da vigilância nas nuvens, a "alimentação" com os dados e atualização da planilha permitirão maior controle e sistematização sobre as informações acerca dos usuários em situação descompensada (DA SILVA LG, et al., 2018). A implantação da planilha traz dinamicidade e efetividade aos serviços, contribuindo para a melhoria do acompanhamento das condições de vida e saúde das famílias, qualificando a gestão dos cuidados e possibilitando a busca ativa dos usuários (DA ROSA LGF, et al., 2017).

O uso de tecnologias vem crescendo em vários âmbitos e na área da saúde está se tornando essencial, uma vez que contribui com a agilidade e segurança para o armazenamento de arquivos, além de ampliar o número de profissionais que podem acessar um prontuário ao mesmo tempo (PINTO LF e ROCHA CMF, 2016). Na vigilância das DCNT à tecnologia permite que dados de pacientes sejam monitorados constantemente de forma segura e que informações sejam adicionadas pelos diversos profissionais de saúde de uma equipe.

Durante a coleta de dados foi possível identificar entraves para o preenchimento adequado e completo da planilha, em virtude de falta de algumas informações nos prontuários. Não foi possível, para grande parte dos usuários, obter informações como o uso de tabaco, dados antropométricos, lesão de órgão alvo, DM e necessidade de autocuidado. As informações sobre autocuidado são importantes, pois a doença ou lesão de órgão alvo podem alterar o funcionamento dos sistemas do corpo humano, trazendo necessidades temporárias ou permanentes (SILVA IJ, et al., 2009).

Esse método de monitoramento é essencial para o acompanhamento prolongado de doenças crônicas e demonstra evidentes melhoras para a promoção e cuidado da saúde, como prevenção e controle de doenças (DA SILVA ILC, 2019). Porém, é necessário ressaltar que se trata de um desafio, pois exige mudança na dinâmica de trabalho dos profissionais de saúde e também de certa tecnologia nas UBS (SOARES CHA e LINHARES MSC, 2008).

Nesse contexto, a Educação Permanente em Saúde (EPS) é essencial, sendo considerada uma política de saúde no Brasil, com o objetivo de transformar as práticas profissionais e organizar o trabalho, conforme as necessidades e dificuldades apresentadas, já que os profissionais podem ser capacitados e orientados quanto ao manejo correto do uso da ferramenta de vigilância nas nuvens (FERREIRA L, et al., 2019).

A experiência da utilização da vigilância nas nuvens apontou para a necessidade do registro adequado dos dados pacientes, além de uma atualização periódica dessas informações, a fim de ser um instrumento de fácil e confiável acesso para o acompanhamento dos usuários estratificados. Recomenda-se a 
continuidade desse processo de trabalho, incluindo os usuários que não puderam ser estratificados. Além disso, orienta-se que equipes, em um número cada vez maior, possam incluir em suas práticas tal instrumento de estratificação com facilidade de acesso para a vigilância em saúde.

Faz-se relevante que iniciativas, como essa, sejam divulgadas no âmbito municipal e estimuladas pela gestão entre os demais profissionais desse âmbito de atenção, a fim de se criarem ferramentas/estratégias para aperfeiçoar o processo de trabalho no âmbito da APS. A qualificação dos processos de trabalho poderá permitir melhorar os cuidados para usuários com condições crônicas de saúde, diminuindo taxas de morbimortalidade, significando avanços em termos de qualidade em saúde de indivíduos acompanhados.

A vigilância nas nuvens, por meio da elaboração de uma planilha on-line, permitiu a estratificação de usuários hipertensos e diabéticos cadastrados na ESF de forma imediata. Assim, trata-se de um recurso viável, de baixo custo e de fácil acesso, em que as informações acerca do usuário podem ser rapidamente registradas, atualizadas e acessadas por todos os profissionais da APS, envolvidos no cuidado. Ações interprofissionais, como essas, proporcionam a melhoria dos cuidados prestados e, portanto, a melhoria dos indicadores de saúde e qualidade de vida da população.

\section{AGRADECIMENTOS}

Agradecemos ao Ministério da Saúde; Universidade Estadual de Montes Claros e Prefeitura de Montes Claros, por meio de suas equipes da ESF; pela parceria e apoio ao programa PET-Saúde/ Interprofissionalidade.

\section{REFERÊNCIAS}

1. ALMEIDA RGS, et al. A interface entre o PET-Saúde/Interprofissionalidade e a Política Nacional de Educação Permanente em Saúde. Saúde Debate, 2019; 43(1): 97-105.

2. BATISTA NA, et al. Educação interprofissional na formação em Saúde a experiência da Universidade Federal de São Paulo, campus Baixada Santista. Interface, 2018; 22(2): 1705-15.

3. BRASIL. Ministério da Saúde, Secretaria de Atenção à Saúde, Departamento de Atenção Básica. Diretrizes para o cuidado das pessoas com doenças crônicas nas redes de atenção à saúde e nas linhas de cuidado prioritárias. Brasília, DF: Ministério da Saúde. 2013.

4. BRASIL. Portaria no 2436, de 21 de setembro de 2017 aprova a Política Nacional de Atenção Básica. Brasília, DF: Ministério da Saúde. 2017.

5. BRASIL. Ministério da Saúde.Política Nacional de Educação Permanente em Saúde: o que se tem produzido para o seu fortalecimento?. Brasília, DF: Ministério da Saúde. 2018.

6. BRASIL. Ministério da Saúde. Construindo caminhos possíveis para a Educação Interprofissional em Saúde nas Instituições de Ensino Superior do Brasil. Brasília, DF: Ministério da Saúde. 2018.

7. DA ROSA LGF, et al. Implantação do processo de Vigilância nas Nuvens na Atenção Primária à Saúde. (Relato de experiência). Universidade Luterana do Brasil, Rio Grande do Sul, Canoas, 2017; 1-2p.

8. DANTAS RCO, RONCALLI AG. Protocolo para indivíduos hipertensos assistidos na Atenção Básica em Saúde. Ciênc. saúde coletiva. 2019; 24(1): 295-306.

9. DA SILVA, ILC. Pôsteres- Área 01- Atenção à Saúde: Redes e modelos. Revista Interdisciplinar Ciências e Saúde, $2019 ; 5(1)$.

10. DA SILVA LG, et al. A importância da planilha "vigilância nas nuvens" no monitoramento de pacientes com condições crônicas em um centro de saúde da comunidade. Revista Interdisciplinar Ciências e Saúde, 2018;254.

11. FERREIRA L, et al. Educação Permanente em Saúde na Atenção Básica: uma revisão integrativa da literatura. Saúde debate, 2019; 43(120): 223-239.

12. GARCIA SO, et al. Integração ensino-serviço: experiência potencializada pelo Programa de Educação pelo Trabalho para a Saúde - Eixo Educação Permanente. Interface, 2019; 23: e180540.

13. MALTA DC, et al. A vigilância e o monitoramento das principais doenças crônicas não transmissíveis no Brasil Pesquisa Nacional de Saúde, 2013. Rev. bras. epidemiol, 2015; 18(2): 3-16.

14. MALTA DC, et al. Doenças crônicas não transmissíveis e a utilização de serviços de saúde: análise da Pesquisa Nacional de Saúde no Brasil. Rev Saude Publica, 2017;51; I 1:(4). 1-10s.

15. MENDES EV. O cuidado das condições crônicas na atenção primária à saúde: o imperativo da consolidação da estratégia da saúde da família. / Eugênio Vilaça Mendes. Brasília: Organização Pan-Americana da Saúde, 2012;512 p.

16. MENDEZ RDR, et al. Estratificação do risco cardiovascular entre hipertensos: Influência de fatores de risco. Rev Bras Enferm, 2018; 71(4):1985-91.

17. MINAS GERAIS. Secretaria De Estado De Saúde De Minas Gerais. Linha Guia de Hipertensão Arterial Sistêmica, Diabetes Mellitus e Doença Renal Crônica. 2013. 
18. OLIVEIRA SX, et al. A importância do escore de risco de Framingham na prevenção das doenças cardiovasculares. Temas em Saúde, 2018; 20(6): 751-770.

19. PEREIRA, MF. Interprofissionalidade e saúde: conexões e fronteiras em transformação. Interface, 2018, 22(2):17536.

20. PINTO LF e ROCHA CMF. Inovações na Atenção Primária em Saúde: o uso de ferramentas de tecnologia de comunicação e informação para apoio à gestão local. Ciência e Saúde Coletiva, 2016; 21(5):1433-1448.

21. REEVES S, et al. A BEME systematic review of the effects of interprofessional education: BEME Guide No. 39. Med Teach, 2016; 38(7):656-68.

22. ROJAS A, et al. Tecnologias disponíveis para acompanhamento do desenvolvimento infantil pelo enfermeiro: revisão integrativa da literatura. Cadernos da Escola de Saúde, 2016; 1(15): 64-80.

23. SANTOS MPR, et al. Qualidade da atenção aos pacientes com Diabetes Mellitus no Programa Mais Médicos, em um município da região metropolitana do Recife (PE), Brasil. Saúde debate, 2020; 44(25): 384-399.

24. SILOCCHI C, JUNGES JR. Equipes de Atenção Primária: Dificuldades no cuidado de pessoas com doenças crônicas não transmissíveis. Trab. educ. saúde, 2017; 15(2):599-615.

25. SILVA IJ, et al. Cuidado, autocuidado e cuidado de si: uma compreensão paradigmática para o cuidado de enfermagem. Rev. esc. enferm, 2009 ; 43( 3 ): 697-703.

26. SILVA RP, et al. A experiência de alunos do PET-Saúde com a saúde indígena e o programa Mais Médicos. Interface, 2015; 19(1):1005-1014.

27. SOARES CHA, LINHARES MSC. Vigilância à saúde no município de Sobral-CE: aspectos históricos, conceituais e atuação na saúde pública. SANARE, 2008; 7(1): 6-11.

28. SOCIEDADE DE CARDIOLOGIA DO ESTADO DO RIO DE JANEIRO. Manual de prevenção cardiovascular. 1 ed. Rio de Janeiro. 2017.

29. STARFIELD B. Atenção primária: equilíbrio entre necessidades de saúde, serviços e tecnologia. 2002.

30. TANAKA OY, et al. Hipertensão arterial como condição traçadora para avaliação do acesso na atenção à saúde. Ciência \& Saúde Coletiva, 2019, 24(3):963-972.

31. UNIVERSIDADE ABERTA DO SUS (UNA-SUS). Núcleo Telessaúde Estadual do Rio Grande do Sul. Curso EAD de Qualificação dos Processos de Trabalho e Gestão da APS: "vigilância nas nuvens", 2017. 